\title{
A uniquely homogeneous space need not be
} a topological group

by

Jan van Mill (Amsterdam)

Abstract. A (separable metric) space $X$ is called uniquely homogeneous provided that for al $x, y \in X$ there is a unique homeomorphism of $X$ taking $x$ onto $y$. Each uniquely homogeneous space $x, y \in X$ there is anich admits a natural group structure for which all $X$ for which this group structure is Abelian, which an example of a uniquely homogeneous space $X$ fomeomorphisms, such that $X$ does not admit the structure of a topological group.

O. Introduction. All spaces under discussion are separable metric. A space $X$ is called uniquely homogeneous provided that for all $x, y \in X$ there is a unique called uniquely homogeneous provided that for and Renaud [1], using a result of Ungar [14], showed that a uniquely homogeneous space cannot be compact, or Ungar [14], showed that a locally connected. The author presented in [9] an example of a connected and locally connected topological group $G$ which is Boolean (each element of the group has order at most 2), and which has the additional property that each autohomeomorphism of $G$ is a translation. An easy consequence of these properties of $G$ is that $G$ is uniquely homogeneous.

Ungar [14] defines a natural group structure on a uniquely homogeneous space $X$ for which it is easily seen that all left translations of $X$ are homeomorphisms, and shows that if $X$ is compact, or locally compact and locally connected, then and shows that if $X$ is com $X$ into a topological group. In addition he showed that the group of topological isomorphisms of $X$ is trivial. Subsequently, Barit and Renaud [1] showed that a locally compact group different from $\{0\}$ or $Z_{2}$ always Renaud [1] showed that a locally compact grous a the result that aniquely homogeneous space containing more than one point cannot be compact, or locally compact and locally connected.

In view of the above remarks and in view of the fact that all known examples of uniquely homogeneous spaces are topological groups, the question naturally arises whether every uniquely homogeneous space admits the structure of a topological group. We will answer this question in the negative by constructing an example of a uniquely homogeneous semitopological group which does not admit the structure of a topological group. This answers a question raised in [9]. 
1. Preliminaries. If $G$ is an Abelian group then we let " + " denote the group operation on $G$. We regard + to be a function of $G \times G$ onto $G$ and for $+(x, y)$ we write $x+y$ of course. If $K \subseteq G \times G$ then the restriction $+\mid K$ will also be denoted by + .

A group is called Boolean if each element of the group has order at most 2. Observe that a Boolean group is Abelian. Let $G$ be a group. If $A \subseteq G$ then «A» denotes the subgroup of $G$ generated by $A$. Let $G$ be a Boolean group and let $H \subseteq G$ be a subgroup. If $x \in G$ then it is easily seen that

$$
\ll H \cup\{x\} »=H \cup(x+H) .
$$

A group $(G,$.$) equipped with a topology is called a semitopological group if$ the operation $: G \times G \rightarrow G$ is continuous in each variable separately. It is known that if $G$ is a semitopological group then $G$ is a topological group if $G$ is Baire, see Husain [5, p. 38].

A cardinal is an initial ordinal and an ordinal is the set of smaller ordinals. c denotes $2^{\text {so }}$.

The domain and range of a function $f$ will be denoted by $\operatorname{dom}(f)$ and $\operatorname{range}(f)$, respectively. Observe that the cardinality of the collection of all $G_{\delta}$-subsets of a given space is at most $c$. This implies that if $X$ and $Y$ are spaces, then the collection has cardinality at most c.

$$
\mathscr{F}=\left\{f: \operatorname{dom}(f) \text { is a } G_{\delta} \text {-subset of } X \text { and } \operatorname{range}(f) \subseteq Y\right\}
$$

Let $X$ be a space. A subset $U \subseteq X$ is called regular open provided that $U$ $=\operatorname{int}_{X} \mathrm{cl}_{X} U$. Let $R O(X)=\{U \subseteq X: U$ is regular open $\}$. It is well-known, and easy to prove (see Sikorski $[13, \S 1.20]$ ), that $R O(X)$ is a complete Boolean algebra under the following operations:

and

$$
\begin{aligned}
& U \wedge V=U \cap V, \\
& U \vee V=\operatorname{int}_{X} \operatorname{cl}_{X}(U \cup V),
\end{aligned}
$$

$$
U^{\prime}=\operatorname{int}_{X}(X \backslash U)
$$

Let $\mathscr{B} \subseteq R O(X)$ be a subalgebra. The Stone space of $\mathscr{B}$, denoted by st $(\mathscr{B})$, has as underlying set the set of all uitrafilters in $\mathscr{B}$. If $U \in \mathscr{B}$ then $\tilde{U}=\{\beta \in \operatorname{st}(\mathscr{B})$ : $U \in \beta\}$. The topology of st $(\mathscr{B})$ is generated by the collection

$$
\{\tilde{U}: U \in \mathscr{B}\} \text {. }
$$

The sets $\tilde{U}$ are clopen ( $\equiv$ closed and open) and st $(\mathscr{B})$ is compact. The reader should observe that if $\mathscr{B}$ is countable then st $(\mathscr{B})$ is metrizable and that then for the definition of $\mathrm{st}(\mathscr{B})$ one does not need the full strength of the axiom of choice. Since $\mathscr{B}$ is countable, if $\gamma \subseteq \mathscr{B}$ is a filter then $\gamma$ can be extended to an ultrafilter $\beta \subseteq \mathscr{O}$ by a process of countably many steps.

If $X$ is a space, then $H(X)$ denotes the group of all autohomeomorphisms. of $X$. The identity of $X$ will be denoted by id.

If $X$ is a space, and if $A \subseteq X$ then $\operatorname{cl}_{X} A$ and $\bar{A}$ denote the closure of $A$ in $X$.
2. Unique homogeneity. In this section we will show that each uniquely homogeneous space admits a very natural group structure such that all left translations are homeomorphisms. The results in this section are included for completeness sake; they are well-known and easy to prove, see Ungar [14].

2.1. LemMA. Let $X$ be homogeneous. The following statements are equivalent:

(1) $X$ is uniquely homogeneous,

(2) if $f, g \in H(X)$ and $f(x)=g(x)$ for certain $x \in X$, then $f=g$,

(3) if $f \in H(X)$ and $f$ has a fixed point, then $f=$ identity.

Proof. (1) $\Rightarrow(3)$. Suppose that $f(x)=x$ for certain $f \in H(X)$ and $x \in X$. Since the identity is a homeomorphism taking $x$ onto $x$, by (1), $f=\mathrm{id}$.

(3) $\Rightarrow(2)$. Let $h=g^{-1} f$. Then $h(x)=x$ and therefore, by (3), $h=$ id. We conclude that $f=g$.

$(2) \Rightarrow(1)$. Take $x, y \in X$. Since $X$ is homogeneous, there is an $f \in H(X)$ with $(x)=y$. By (2) this $f$ is unique.

Let $X$ be uniquely homogeneous. Fix $e \in X$ and for each $x \in X$ let $f_{x}$ be the unique homeomorphism taking $e$ onto $x$. Define a binary operation " + " and an operation "-" on $X$ by

$$
x+y=f_{x}(y), \text { and }-x=f_{x}^{-1}(e) .
$$

2.2. Lemma. Let $x, y, z \in X$. Then

(1) $x+(y+z)=(x+y)+z$,

(2) $x+e=e+x=x$,

(3) $x+(-x)=(-x)+x=e$.

Proof. (1) Observe that

$$
\left(f_{x} f_{y}\right)(e)=f_{x}\left(f_{y}(e)\right)=f_{x}(y)=x+y .
$$

and

$$
f_{x+y}(e)=x+y .
$$

By Lemma 2.1(2) we therefore conclude that $f_{x} f_{y}=f_{x+y}$. Since

$$
x+(y+z)=x+f_{y}(z)=f_{x}\left(f_{y}(z)\right)=\left(f_{x} f_{y}\right)(z) \text { and }(x+y)+z=f_{x+y}(z),
$$

we conclude that $x+(y+z)=(x+y)+z$.
(2) trivial.

(3) $x+(-x)=f_{x}\left(f_{x}^{-1}(e)\right)=e$. Notice that $f_{-x}(e)=-x=f_{x}^{-1}(e)$. Lemma $2.1(2)$ therefore implies that $f_{-x}=f_{x}^{-1}$. Consequently, $(-x)+x=f_{-x}(x)$
$=f_{x}^{-1}(x)=e$.

We conclude that + is a very natural group operation on $X$.

2.3. Lemma. All left translations of $X$ are homeomorphisms.

Proof. Fix $a \in X$ and define $l_{a}: X \rightarrow X$ by $l_{a}(x)=a+x$. Then $l_{a}(x)=f_{a}(x)$ for all $x \in X$. We conclude that $l_{a}=f_{a}$. 
3. A theorem. The aim of this section is to prove Theorem 3.1 below, which is the key in the construction of our example. The proof of this result is similar to the proof of Theorem 3.1 in [9] except for some minor changes. Since these changes are not always obvious, we will give the proof in full detail.

3.1. THEOREM. Let $X$ be a Boolean semitopological group with the following properties

(a) $X=\bigcup_{n=1}^{\infty} X_{n}$, where each $X_{n}$ is a topologically complete subgroup of $X$, and $X_{n} \subseteq X_{n+1}$ for all $\dot{n} \in N$,

(b) if $x, y \in X$ then there exist an $n \in N$ and a disc $D \subseteq X_{n}$ containing both $x$ and $y$.

If $E \subseteq X$ is a countable subgroup, then there is a dense subgroup $H \subseteq X$ containing $E$ such that the autohomeomorphisms of $H$ are precisely its translations. Consequently, $H$ is uniquely homogeneous.

Proof. Let $a X$ be a compactification of $X$, Let $\mathscr{F}=\{f: \operatorname{dom}(f)$ and range $(f)$ are $G_{\delta}$-subsets of $\left.a X\right\}$. Since $|\mathscr{F}| \leqslant c$, we can enumerate $\mathscr{F}$ by $\left\{f_{\alpha}: \alpha<c, \alpha\right.$ even $\}$, Let $\left\{K_{\alpha}: \alpha<\mathfrak{c}, \alpha\right.$ odd $\}$ enumerate all Cantor sets in $X$. By transfinite induction, for every $\alpha<c$ we will construct subgroups $H_{\alpha} \subseteq X$ and subsets $V_{\alpha} \subseteq a X$ such that

(1) if $\beta<\alpha$ then $E \subseteq H_{\beta} \subseteq H_{\alpha}, V_{\beta} \subseteq V_{\alpha}$ and $H_{\alpha} \cap V_{\alpha}=\varnothing$,

(2) $\left|H_{\alpha}\right| \leqslant|\alpha| \cdot \kappa_{0}$ and $\left|V_{\alpha}\right| \leqslant|\alpha| \cdot s_{0}$,

(3) if $\alpha$ is odd then $H_{\alpha} \cap K_{\alpha} \neq \varnothing$,

(4) if $\alpha$ is even and if $\left|\left\{x \in \operatorname{dom}\left(f_{\alpha}\right) \cap X: f_{\alpha}(x) \notin \ll \bigcup_{\beta<\alpha} H_{\beta} \cup\{x\} »\right\}\right|=c$; then there is a point $x \in \operatorname{dom}\left(f_{\alpha}\right) \cap H_{\alpha}$ such that $f_{\alpha}(x) \in V_{\alpha}$.

Suppose that we have completed the construction for all $\beta<\alpha$, where $\alpha<\mathrm{c}$. For convenience, put $H^{\alpha}=\bigcup_{\beta<\alpha} H_{\beta}$ and $V^{\alpha}=\bigcup_{\beta<\alpha} V_{\beta} \cap X$. Observe that $\left|H^{\alpha}\right|$ $\leqslant|\alpha| \cdot \kappa_{0}<\mathrm{c}$, and, similarly, that $\left|V^{x}\right| \leqslant|\alpha| \cdot \kappa_{0}<\mathrm{c}$.

Case $1 . \alpha$ is odd. Since $\left|K_{\alpha}\right|=c$, we can pick a point

$$
x \in K_{\alpha} \backslash\left(H^{\alpha}+V^{\alpha}\right) \text {. }
$$

Define $H_{\alpha}=\left\langle H_{\alpha} \cup\{x\} »\right.$ and $V_{\alpha}=\bigcup_{\beta<\alpha} V_{\beta}$. An easy check shows that $H_{\alpha}$ and $V_{\alpha}$ are as required.

Case 2. $\alpha$ is even and $|S|<$ c where $S=\left\{x \in \operatorname{dom}\left(f_{\alpha}\right) \cap X: f_{\alpha}(x) \notin \ll H^{\alpha} \cup\{x\} »\right\}$. Define $H_{\alpha}=H^{\alpha}$ and $V_{\alpha}=\bigcup V_{\beta}$.

Case 3. $\alpha$ is even and $|S|=$ c. By the same argument as in Case 1, we can find a point

$$
x \in S \backslash\left(H^{\alpha}+V^{\alpha}\right)
$$

Define $H_{\alpha}=\ll H^{\alpha} \cup\{x\} »$ and $V_{\alpha}=\bigcup_{\beta<\alpha} V_{\beta} \cup\left\{f_{\alpha}(x)\right\}$. Since

$$
H_{\alpha} \cap V_{\alpha}=\left(H^{\alpha} \cup\left(x+H^{\alpha}\right)\right) \cap\left(\bigcup_{\beta<\alpha} V_{\beta} \cup\left\{f_{\alpha}(x)\right\}\right)=\varnothing,
$$

we see' that $H_{\alpha}$ and $V_{\alpha}$ are as required.

Now put $H=\bigcup H_{\alpha}$. We claim that $H$ is as required. Let $f: H \rightarrow H$ be a homeomorphism. Our task is to find a point $h \in H$ such that $f(x)=x+h$ for all $x \in H$.

By the well-known Lavrentieff Theorem ([6]; see also [3, Thm. 4.3.21]), there are $G_{\delta}$-subsets $S$ and $T$ of $a X$ such that $f$ can be extended to a homeomorphism $f: S \rightarrow T$. Let $\alpha<c$ be such that $f=f_{\alpha}$.

Case 1. $\left|\left\{x \in S \cap X: f_{\alpha}(x) \notin \ll \bigcup_{\beta<\alpha} H_{\beta} \cup\{x\} »\right\}\right|=c$. Then by (4), there is a point $x \in S \cap H$ such that $f_{\alpha}(x) \notin H$. Since $f_{\alpha}$ extends $f$, this is impossible.

Case 2. Not Case 1. Let

$$
H^{\alpha}=\bigcup_{\beta<\alpha} H_{\beta} \quad \text { and } \quad U=\left\{x \in S \cap X: f_{\alpha}(x) \notin \bigcup_{\beta<\alpha} H_{\beta} \cup\{x\} »\right\} .
$$

By assumption, $|U|<c$. For each $h \in H^{\alpha}$ define

$$
E_{h}=\left\{x \in S \cap X: f_{\alpha}(x)=x+h\right\} .
$$

We claim that $E_{h}$ is closed in $S$. For all $n \in N$ take $x_{n} \in E_{h}$ and $x \in S$ such that $\lim x_{n}=x$. Then

$\lim _{n \rightarrow \infty}$

$$
f_{\alpha}(x)=\lim _{n \rightarrow \infty} f_{\alpha}\left(x_{n}\right)=\lim _{n \rightarrow \infty}\left(x_{n}+h\right)=x+h
$$

(this uses of course only the fact that $X$ is semitopological). In addition the collection $\mathscr{E}=\left\{E_{h}: h \in H^{\alpha}\right\}$ is clearly pairwise disjoint. For each $h \in H^{\alpha}$ let $F_{h}$ $=E_{h} \backslash f_{\alpha}^{-1}\left(H^{\alpha}\right)$. We conclude that the collection $\mathscr{G}=\left\{F_{h}: h \in H^{\alpha}\right\}$ is also pairwise disjoint. We claim that at most one set of the collection $\mathscr{G}$ can be nonempty. To the contrary, suppose that there exist distinct points $s, t \in H^{\alpha}$ and points $x \in F_{s}$ and $y \in F_{t}$. We first claim that $X \backslash S$ is countable. If not, then there is an $n \in N$ such that $X_{n} \backslash S$ is uncountable. Since $X_{n} \backslash S$ is a countable union of closed subsets of $X_{n}$, one of these closed sets must be uncountable and therefore contains a Cantor set since $X_{n}$ is topologically complete, [4] (see also [3, 4.5.5]). This is impossible since $H \subseteq S$ intersects all Cantor sets. Find an $n \in N$ and a disc $D \subseteq X_{n}$ containing both $x$ and $y$. Since $|U|<c$ and since $\left|f^{-1}\left(H^{\alpha}\right)\right|<c$, we can choose an arc $J \subseteq D$ which connects $x$ and $y$ but misses $U \cup f^{-1}\left(H^{\alpha}\right) \cup X \backslash S$. We conclude that $J \subseteq \cup \mathscr{G}$. Put $K=\left\{h \in H^{\alpha}: F_{h} \cap J \neq \varnothing\right\}$. By assumption, $|K| \geqslant 2$, whence $\left\{J \cap E_{h}: h \in K\right\}$ is a partition of $J$ in at least 2 closed and disjoint, nonempty sets. Notice that $E_{h} \cap J$ is closed in $J$ since $J \subseteq S$. By Sierpiński's Theorem, [11] (see also [3, Thm. 5.3.2]), $|K|>\aleph_{0}$. Also, $|K| \leqslant\left|H^{\alpha}\right|<\mathrm{c}$ (so in case the Continuum Hypothesis holds, we have derived a contradiction). Find an $m \geqslant n$ such that $K \cap X_{m}$ is uncountable. Then $K \cap X_{m}$ is not closed in $X_{m}$ since $X_{m}$ is topologically complete and $\aleph_{0}$ 
$<\left|K \cap X_{m}\right|<c$. Let $k_{i} \in K \cap X_{m}(i \in N)$ be a sequence converging to a point $k \in X_{m} \backslash K$. For each $i \in N$, take a point $x_{i} \in J \cap F_{k_{i}}$. By compactness of $J$, we may assume that $\lim _{i \rightarrow \infty} x_{i}=p \in J$. Since $X_{m}$ is a semitopological group which is topo$\lim _{i \rightarrow \infty}$

logically complete, by Montgomery [10] (see also [5, p. 38]) $X_{m}$ is a topological group with group operation + . We conclude that $+: X_{m} \times X_{m} \rightarrow X_{m}$ is continuous. Consequently,

$$
f_{\alpha}(p)=\lim _{i \rightarrow \infty} f_{\alpha}\left(x_{i}\right)=\lim _{i \rightarrow \infty}\left(x_{i}+k_{i}\right)=p+k
$$

(notice that $x_{i}, k_{i} \in X_{m}$ for all $i \in N$ ). Since $p \in J$, there is an $h \in K$ such that $p \in E_{h}$. Therefore,

$$
f_{\alpha}(p)=p+h=p+k,
$$

which implies that $h=k$, a contradiction.

If for each $h \in H^{\alpha}$ it is true that $F_{h}=\varnothing$, then $f_{\alpha}(H) \subseteq f_{\alpha}(U) \cup H^{\alpha}$. Since $H$ intersects all Cantor sets in $X$, the cardinality of $H$ must be c. Since $f_{\alpha}$ is one-to-one, this implies that $f_{\alpha}(H)$ has cardinality c. However, since $f_{\alpha}(U) \cup H^{\alpha}$ has cardinality less than c, this is impossible. Consequently, there is precisely one $h \in H^{\alpha}$ for which $F_{h} \neq \varnothing$. Since $H$ intersects all Cantor sets in $X$, each nonempty open subset of $H$ has clearly cardinality $c$. Since $E_{h}$ is closed in $S$, and since $S \backslash E_{h} \subseteq U$ has cardinality less than $\mathrm{c}$, we may conclude that $E_{h}=S$. This proves that $f_{\alpha}$ is a translation.

3.2. Remark. In the proof of the above result we used a technique originally due to Kuratowski [7]. Some specific ideas needed for the proof can be found in [8], and also in [9]. For more references and information concerning Kuratowski's technique, see [9]

4. A Boolean group structure on $Q$. Let $Q$ denote the space of rational numbers. Among other things, we will show that there is a Boolean group structure * on $Q$ such that all translations are (topological) homeomorphisms while moreover the function $*: Q \times Q \rightarrow Q$ is nowhere continuous $\left({ }^{1}\right)$.

Let $K=\{0,1)^{N}$ and let $G$ be a countable dense subgroup of $K$. Observe that $G$ is Boolean. For all $n \in N$, define

$$
A_{n}=\left\{x \in K: x_{i}=0 \text { if } i \in\{1,2, \ldots, n-1, n+1, \ldots, 2 n-1\} \text { and } x_{n}=1\right\} .
$$

It is clear that $A_{n}$ is clopen, that the diameter of $A_{n}$ tends to 0 if $n$ goes to infinity, and that each neighborhood of the identity of $K$, which we will denote by $O_{K}$, contains all but finitely many of the $A_{n}$ 's. Let $A=\bigcup_{n=1}^{\infty} A_{n}$. Observe that $A \in R O(K)$.

$$
\text { 4.1. LeMMA. If } n<m<2 n \text { then }\left(A_{n}+A_{m}\right) \cap \bar{A}=\varnothing \text {. }
$$

Proof. If $x \in A_{n}$ and $y \in A_{m}$ then $(x+y)_{i}=0$ if $i \leqslant n-1,(x+y)_{n}=1$ and there is a $j \in N$ with $n<j \leqslant 2 n-1$ such that $(x+y)_{j}=1$. This easily implies that $x+y \notin \bar{A}$.

(1) It is well-known of course that a semitopological group need not be a topological group. In our construction we need a Boolean group structure as above. I do not know whether this has been done before.
4.2. LEMMA. If $F \subseteq N$ is finite and if $N \backslash F=\bigcup_{i=1}^{k} B_{i}$ then there is an $i \leqslant k$ such that $B_{i}$ contains two points $n$ and $m$ such that $n<m<2 n$.

Proof. Choose $r \in N$ such that $r>\max F$ and $r>k$. There are $x, y \in N$ with $r \leqslant x<y<2 r$ and there is an $i \leqslant k$ with $\{x, y\} \subseteq B_{i}$. It is clear that $x<y<2 x$.

4.3. Proposition. If $\mathscr{E}$ is a finite family of subsets of $A$ such that for all $E \in \mathscr{E}$ we have that $E+E \subseteq \bar{A}$, then there are infinitely many $n \in N$ with $A_{n} \cap \cup \mathscr{E}=\varnothing$.

Proof. Suppose that $F=\left\{n \in N: A_{n} \cap \cup \mathscr{E}=\varnothing\right\}$ is finite. By Lemma 4.2 there is an $E \in \mathscr{E}$ and there are $n, m \in N \backslash F$ such that $n<m<2 n$ and $E \cap A_{n} \neq \varnothing$ $\neq A_{m} \cap E$. Since by assumption $E+E \subseteq A$, this contradicts Lemma 4.1.

4.4. LEMMA. There is a countable subalgebra $\mathscr{B} \subseteq R O(K)$ such that

(1) if $C \subseteq K$ is clopen then $C \in \mathscr{B}$,

(2) $A \in \mathscr{B}$,

(3) if $x \in G$ and $B \in \mathscr{B}$ then $x+B \in \mathscr{B}$.

Proof. Let $\mathscr{B}_{0}$ be the smallest subalgebra of $R O(K)$ containing all clopen subsets of $K$ and the set $A$. Observe that $\mathscr{B}_{0}$ is countable. Inductively construct countable subalgebras $\mathscr{B}_{n} \subseteq R O(K)$ such that for all $n \in N$,

(a) $\mathscr{B}_{n-1} \subseteq \mathscr{B}_{n}$,

(b) if $x \in \mathscr{B}_{n-1}$ and $x \in G$ then $x+B \in \mathscr{B}_{n}$.

Suppose that the subalgebras are constructed for all $i \leqslant n$. Let $\mathscr{B}_{n+1}$ be the smallest subalgebra of $R O(K)$ containing the collection $\left\{x+B: x \in G\right.$ and $\left.B \in \mathscr{B}_{n}\right\}$. It is clear that $\mathscr{B}_{n+1}$ is countable.

Now put $\mathscr{B}=\bigcup_{n=0}^{\infty} \mathscr{B}_{n}$. An easy check shows that $\mathscr{B}$ is as required.

For each $x \in G$ define $f_{x}: K \rightarrow K$ by $f_{x}(t)=x+t$. Let $X=\operatorname{st}(\mathscr{B})$. Define $\varphi_{x}: X \rightarrow X$ by $\varphi_{x}(\beta)=\left\{f_{x}(B): B \in \beta\right\}$. By Stone duality, $\varphi_{x}$ is a homeomorphism. Consider the following subcollection of $\mathscr{B}$ :

$\gamma=\left\{B^{\prime}: B \in \mathscr{B}, B \subseteq A\right.$ and $\left.B+B \subseteq \bar{A}\right\} \cup\left\{C \subseteq K: C\right.$ is clopen and $\left.O_{K} \in C\right\} \cup\{A\}$.

4.5. LEMMA. If $\mathscr{E} \subseteq \gamma$ is finite, then $\bigwedge \mathscr{E} \neq 0$.

Proof. Without loss of generality, $\mathscr{E}=\left\{B_{1}^{\prime}, \ldots, B_{m}^{\prime}\right\} \cup\{A\} \cup\{C\}$, where for each $i \leqslant m$ we have that $B_{i} \cup\left(B_{i}+B_{i}\right) \subseteq \bar{A}$, and $C$ is a clopen neighborhood of $O_{K}$. By Proposition 4.3, the set $\left\{n \in N: A_{n} \cap \bigcup_{i=1}^{m} B_{i}=\varnothing\right\}$ is infinite. Since $C$ contains almost all of the $A_{n}{ }^{\prime}$ s, we conclude that $\bigwedge \mathscr{E} \neq \varnothing$

By the above Lemma we can extend the collection $\gamma$ to an ultrafilter $\beta \subseteq \mathscr{B}$. Whence $\beta \in \operatorname{st}(\mathscr{B})=X$. Define

$$
Y=\left\{\varphi_{x}(\beta): x \in G\right\} .
$$

We topologize $Y$ by regarding it to be a subspace of $X$.

4.6. LeMMA. If $\varphi_{x}(\beta)=\varphi_{y}(\beta)$ then $x=y$. 
Proof. Suppose that $x \neq y$. Find a clopen neighborhood $C$ of $O_{K}$ such that $(x+C) \cap(y+C)=\varnothing$. Since $x+C=f_{x}(C) \in \varphi_{x}(\beta)$ and $y+C=f_{y}(C) \in \varphi_{y}(\beta)$, we find that $\varphi_{x}(\beta) \neq \varphi_{y}(\beta)$, which is a contradiction.

Define a binary operation $*$ on $Y$ by $\varphi_{x}(\beta) * \varphi_{y}(\beta)=\varphi_{x+y}(\beta)$. By Lemma 4.6, the operation $*$ is well defined. For notational simplicity, the point $\varphi_{x}(\beta)$ will be denoted by $t_{x}$ from now on.

4.7. LeMMA. $(Y, *)$ is a Boolean group with identity $\beta$. In addition, all translations of $Y$ are (topological) homeomorphisms of $Y$.

Proof. That $(Y, *)$ is a Boolean group with identity $\beta$ is trivial. Take $t_{x} \in Y$ and consider the translation $\xi\left(t_{y}\right)=t_{x} * t_{y}$. Observe that

$$
\xi\left(t_{y}\right)=t_{x+y}=\varphi_{x+y}(\beta)=\varphi_{x}\left(\varphi_{y}(\beta)\right)=\varphi_{x}\left(t_{y}\right)
$$

for all $y \in Y$. Consequently, $\xi=\varphi_{x} \mid Y$. Since by construction $\varphi_{x} \mid Y$ is an autohomeomorphism of $Y$, we conclude that $\xi$ is an autohomeomorphism of $Y$.

It is easily seen that $X$ has no isolated points. Since $Y$ is dense in $X$, we conclude that $Y \approx Q$, Sierpiński [12].

4.8. THEOREM. There is a Boolean group structure $*$ on $Q$ such that

(1) $*: Q \times Q \rightarrow Q$ is nowhere continuous,

(2) all translations of $Q$ are (topological) homeomorphisms.

Proof. It clearly suffices to show that $*: Y \times Y \rightarrow Y$ is not continuous in $(\beta, \beta)$. We claim that $*^{-1}(\tilde{A})$ is not a neighborhood of $(\beta, \beta)$ in $Y \times Y$. Indeed, to the contrary assume that there is a neighborhood $\widetilde{B}$ of $\beta$ in $X$, where $B \in \mathscr{B}$, such that

$$
*((\widetilde{B} \cap Y) \times(\widetilde{B} \cap Y)) \subseteq \widetilde{A} .
$$

It is clear that without loss of generality we may assume that $B \subseteq A$. Take $x, y$ $\in B \cap G$. Then $B$ is a neighborhood of both $x$ and $y$, so

$$
\left(t_{x}, t_{y}\right) \in(\widetilde{B} \cap Y) \times(\widetilde{B} \cap Y)
$$

and consequently, $t_{x} * t_{y} \in \tilde{A}$ or, equivalently, $\varphi_{x+y}(\beta) \in \tilde{A}$. Since $\varphi_{x+y}(\beta)$ $=\{(x+y)+E: E \in \beta\}$, there is an $E \in \beta$ such that $(x+y)+E=A$. Since $O_{K} \in \bar{E}$ this implies that $(x+y)+O_{K} \in \bar{A}$. We conclude that $x+y \in \bar{A}$. Since $B \cap G$ is dense in $B$, we therefore have that $B+B \subseteq \bar{A}$. By definition of $\gamma, B^{\prime} \in \gamma \subseteq \beta$. Since by assumption $B \in \beta$, this is a contradiction.

5. Spaces of measurable functions. Let $X$ be a space. A function $f:[0,1] \rightarrow X$ is said to be measurable if $f^{-1}(U)$ is a Borel subset of $[0,1]$ for every open subset $U \subseteq X$. Measurable functions $f, g:[0,1] \rightarrow X$ are called equivalent if

$$
\lambda(\{t \in[0,1]: f(t) \neq g(t)\})=0,
$$

where $\lambda$ denotes Lebesgue measure on $[0,1]$. Let $M_{X}$ be the topological space of equivalence classes of measurable functions from $[0,1]$ into $X$ with the topology of convergence in measure. The topology of $M_{X}$ is determined by the metric

$$
\varrho(f, g)=\left(\int_{0}^{1}(d(f(t), g(t)))^{2} d t\right)^{1 / 2},
$$

where $d$ is any bounded metric compatible with the topology of $X$. The topology of $M_{X}$ does not depend on the chosen metric $d$ on $X$. Bessaga and Pełczyński [2] show that $M_{X} \approx l_{2}$, the separable Hilbert space, if and only if $X$ is completely metrizable and contains more than one point. $\mathrm{It}$ is easily seen that the set of constant functions is closed in $M_{X}$ and isometric to $X$. We identify $X$ and this isometric copy of $X$ in $M_{X}$.

Let $*$ be the Boolean group structure on $Q$ given by Theorem 4.8. Define a Boolean group structure, which we will also denote by $*$, on $M_{Q}$ as follows:

$$
(f * g)(t)=f(t) * g(t) .
$$

It is trivial to verify that $*$ is indeed a Boolean group structure on $M_{Q}$ and that $Q$ is a subgroup of $M_{Q}$. It is also easily seen that all translations of $M_{Q}$ are homeomorphisms, whence $M_{Q}$ is a semitopological group which is not a topological group since $Q$ is a subgroup, see Bessaga and Pełczyński [2]. Define

$$
X=\left\{f \in M_{Q}: f([0,1]) \text { is finite }\right\} \text {. }
$$

5.1. LemMa, $X$ is a subgroup of $M_{Q}$ and $Q \subseteq X$.

Proof. If $f, g \in X$ then $(f * g)([0,1])$ is contained in the subgroup generated by $f([0,1])$ and $g([0,1])$. This subgroup is clearly finite since $Q$ is a Boolean group. That $Q \subseteq X$ is trivial.

5.2. LEMMA. If $F \subseteq Q$ is a finite subgroup, then $B(F)=\left\{f \in M_{Q}: f([0,1]) \subseteq F\right\}$ is a subgroup of $M_{Q}$ which is homeomorphic to $M_{F}$.

Proof. Obvious.

We will identify $B(F)$ and $M_{F}$

5.3. LEMMA. $X=\bigcup_{n=1}^{\infty} X_{n}$, where $X_{n}$ is a topologically complete subgroup of $X$ and $X_{n} \subseteq X_{n+1}$ for all $n \in N$. Moreover, if $x, y \in X$ then there are an $n \in N$ and a disc $D \subseteq X_{n}$ containing both $x$ and $y$.

Proof. Write $Q$ as $\bigcup_{n=1}^{\infty} F_{n}$, where each $F_{n}$ is a finite subgroup of $Q$ and $F_{n}$ $\subseteq F_{n+1}$ for all $n \in N$. Then $X=\bigcup_{n=1}^{\infty} B\left(F_{n}\right)$. Since $B\left(F_{n}\right) \approx M_{F_{n}} \approx l_{2}$, seo Bessaga and Pełczyński [2], we see that if $X_{n}=B\left(F_{n}\right)$ then $X_{n}$ is as required, Lemma 5.2.

We conclude that the semitopological group $X$ satisfies the conditions of Theorem 3.1. Therefore, $X$ contains a dense subgroup $H$ which contains $Q$ such that all autohomeomorphisms of $H$ are translations. Because of this, it easily follows that $H$ is uniquely homogeneous. 
5.4. THEOREM. $H$ is a uniquely homogeneous semitopological group but $H$ does not admit the structure of a topological group.

Pro of. To the contrary, assume that " $\mathrm{o}$ " is a topological group structure on $H$. If $f: H \rightarrow H$ is defined by $f(x)=x^{-1}$ then $f$ is a homeomorphism having a fixed point. We conclude that $f$ is the identity, Lemma 2.1(3). Therefore, $(H, 0)$ is Boolean. Without loss of generality we may assume that the identities of $(H, \circ)$ and $(H, *)$ are both equal to the same point $e \in H$. Take $x \in H$ arbitrarily. The translation $f(t)=x \circ t$ maps $e$ onto $x$. However, there is only one homeomorphism mapping $e$ onto $x$, namely the translation $g(t)=x * t$. We conclude that for all $t \in H$ we have that $x \circ t=x * t$. Since $x$ was arbitrary, we find that $\circ=*$. However, $*: H \times H \rightarrow H$ is not continuous since by construction $*: Q \times Q \rightarrow Q$ is not continuous. Contradiction.

\section{References}

[1] W. Barit and P. Renaud, There are no uniquely homogeneous spaces, Proc. Amer. Math. Soc. 68 (1978), pp. 385-386.

[2] C. Bessaga and A. Pełczyński, On spaces of measurable functions, Studia Math. 44 (1972), pp. $597-615$.

[3] R. Engelking, General Topology, Warszawa 1977.

[4] F. Hausdorff, Grundzïge der Mengenlehre, Leipzig 1914.

[5] T. Husain, Introduction to topological groups, W. B. Saunders Company, 1960.

[6] M. Lavrentieff, Contribution à la théorie des ensembles homéomorphes, Fund. Math. 8 (1925), pp. 201-208.

[7] K. Kuratowski, Sur la puissance de l'ensemble des „nombres de dimension” de M. Fréchet, Fund. Math. 34 (1925), pp. 201-208.

[8] J. van Mill, Representing countable groups by homeomorphism groups in Hilbert space, Math. Ann. 259 (1982), pp. 321-329.

[9] - A topological group having no homeomorphisms other than translations, Trans. Amer. Math. Soc. 280 (1983), pp. 491-498.

[10] D. Montgomery, Continuity in topological groups, Bull. Amer. Math. Soc. 42 (1936), pp. 879-882.

[11] W. Sierpiński, Un théorème sur les continus, Tôhoku Math. J. 13 (1918), pp. 300-303.

[12] - Sur une propriété topologique des ensembles denombrables denses en soi, Fund. Math. 1 (1920), pp. 11-16.

[13] R. Sikorski, Boolean Algebras, New York 1964.

[14] G. Ungar, On all kinds of homogeneous spaces, Trans. Amer. Math. Soc. 212 (1975), pp. 393-400.

SUBFACULTEIT WISKUNDE

VRIJE UNIVERSITEIT

De Boelelaan 1081

Amsterdam

The Netherlands
MATHEMATISCH INSTITUUT

UNIVERSITEIT VAN AMSTERDAM

Roeterstraat 15

Amsterdam

The Netherland: 